\title{
Pressure-Overload Cardiac Hypertrophy Is Associated with Distinct Alternative Splicing Due to Altered Expression of Splicing Factors
}

\author{
Taeyong Kim, Jin Ock Kim, Jae Gyun Oh ${ }^{1}$, Seong-Eui Hong ${ }^{*}$, and Do Han Kim*
}

\begin{abstract}
Chronic pressure-overload cardiac hypertrophy is associated with an increased risk of morbidity/mortality, largely due to maladaptive remodeling and dilatation that progresses to dilated cardiomyopathy. Alternative splicing is an important biological mechanism that generates proteomic complexity and diversity. The recent development of next-generation RNA sequencing has improved our understanding of the qualitative signatures associated with alternative splicing in various biological conditions. However, the role of alternative splicing in cardiac hypertrophy is yet unknown. The present study employed RNA-Seq and a bioinformatic approach to detect the RNA splicing regulatory elements involved in alternative splicing during pressure-overload cardiac hypertrophy. We found GC-rich exonic motifs that regulate intron retention in $5^{\prime}$ UTRs and AT-rich exonic motifs that are involved in exclusion of the AT-rich elements that cause mRNA instability in $3^{\prime}$ UTRs. We also identified motifs in the intronic regions involved in exon exclusion and inclusion, which predicted splicing factors that bind to these motifs. We found, through Western blotting, that the expression levels of three splicing factors, ESRP1, PTB and SF2/ASF, were significantly altered during cardiac hypertrophy. Collectively, the present results suggest that chronic pressure-overload hypertrophy is closely associated with distinct alternative splicing due to altered expression of splicing factors.
\end{abstract}

\section{INTRODUCTION}

Cardiac hypertrophy is an adaptive response to various stress conditions such as pressure-overload. Untreated pathological cardiac hypertrophy is associated with an increased risk of

School of Life Sciences and Systems Biology Research Center, Gwangju Institute of Science and Technology, Gwangju 500-712, Korea, ${ }^{1}$ Cardiovascular Research Center, Icahn School of Medicine at Mount Sinai, New York, 10029, USA

*Correspondence: dhkim@ gist.ac.kr (DHK); hse354@ gist.ac.kr (SEH)

Received 14 November, 2013; accepted 18 November, 2013; published online 27 January, 2014

Keywords: cardiac hypertrophy, ESRP1, PTB, RNA-Seq, SF2/ASF, splicing factor morbidity and mortality, largely due to maladaptive remodeling and dilatation that progresses to dilated cardiomyopathy and heart failure.

Alternative splicing is a key biological event that contributes to proteome diversity and complexity by generating distinct mRNAs in different circumstances, which can influence gene expression. Aberrant splicing can have serious pathological consequences (Kim et al., 2008; Zhou et al., 2012). Our understanding of transcriptomic signatures, including qualitative changes due to alternative splicing, have been vastly increased by the recent development of next-generation RNA sequencing (RNA-Seq) methods. RNA-Seq on various cancers has helped improve the strategies for diagnosis and treatment (Eswaran et al., 2013; Kalari et al., 2012; Ren et al., 2012). Although highthroughput analyses of alternative splicing in cardiac diseases have recently been performed (Ames et al., 2013; Park et al., 2011; Song et al., 2012), the detailed mechanisms of altered alternative splicing during cardiac hypertrophy have not yet been reported.

In the present study, we performed an in silico analysis to detect the binding motifs of splicing factors that regulate alternative splicing during pressure-overload cardiac hypertrophy using RNA-Seq datasets. Through this analysis, we found GCrich exonic motif-mediated intron retention in $5^{\prime}$ untranslated regions (UTRs) and AT-rich exonic motif-mediated exclusion of AREs that conferred mRNA instability in $3^{\prime}$ UTRs. We also identified 20 intronic motifs with a strong positional bias in the introns surrounding variant exons, and experimentally confirmed the differential expression of three splicing factors corresponding to particular motifs. Collectively, our study provides the first report of the unique mechanisms of alternative splicing controlled by various regulatory elements, and identifies the splicing factors that are associated with cardiac hypertrophy.

\section{MATERIALS AND METHODS}

Collection of exon variants and non-regulated exons Our previous RNA-Seq datasets for cardiac hypertrophy were utilized (Song et al., 2012). Exon variants were analyzed based on RefSeq structure and less expressed exons $<10$ reads per kilobase per million reads (RPKM) were discarded. Fisher's exact test $(p<0.1)$ and Bayesian error rate (Vencio et al., 2004) (e $\leq 0.1)$ were used to determine the exon variants. Nonregulated exons were decided by $p>0.5$. 
A

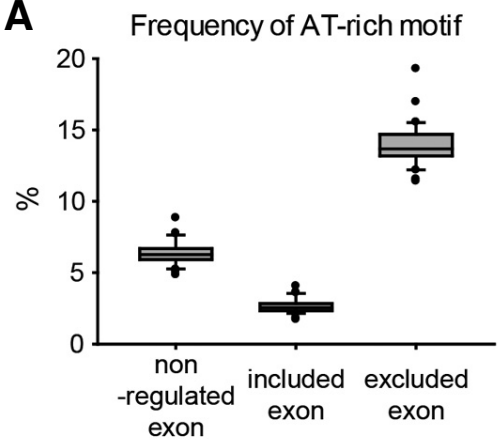

C

$\%$ of AT-rich exons (excluded exons) containing each UTR

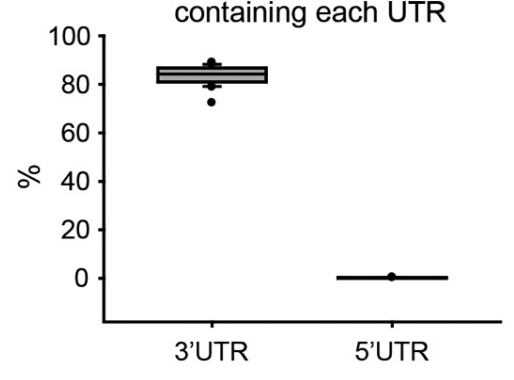

B Frequency of GC-rich motif

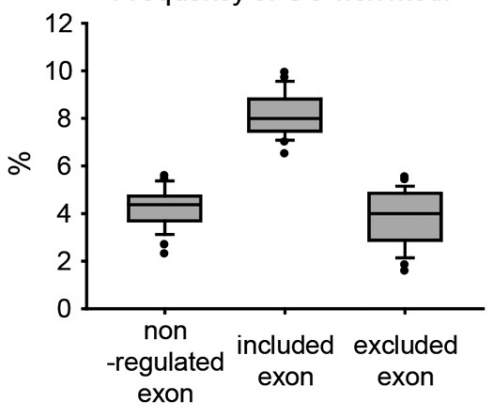

D

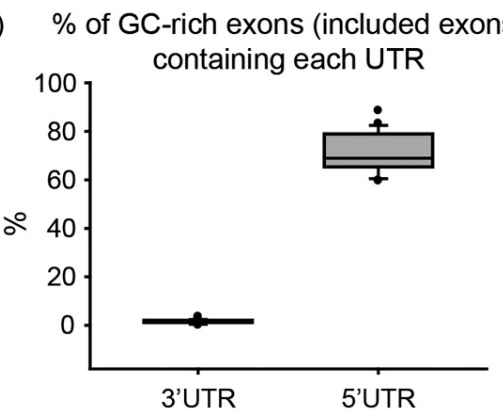

Fig. 1. Characterization of exon variants associated with pressure-overload cardiac hypertrophy. (A) Box plots showing the frequency (\%) of AT-rich motifs in exon variants and non-regulated exons. (B) Box plots showing the frequency (\%) of GC-rich motifs in exon variants and non-regulated exons. (C) Box plots showing the percentage of AT-rich-excluded exons containing $3^{\prime}$ or $5^{\prime}$ UTRs. (D) Box plots showing the percentage of GC-rich-included exons containing $3^{\prime}$ or $5^{\prime}$ UTRs.

\section{Position-specific motif enrichment test}

Given exon variants, $500 \mathrm{bp}$ intronic sequences (R1-R4), defined as Fig. 2A, were collected. If the intron size was smaller than $500 \mathrm{bp}$, whole intron was accepted. Considering the previously reported approaches for examining the regulatory motifs by enriched hexanucleotides in a position-dependent manner (Erkelenz et al., 2013; Fairbrother et al., 2002; Hirose et al., 2006; Huh and Hynes, 1994; Kuroyanagi et al., 2013; Lim et al., 2011; Yamashita et al., 2012), we counted the occurrence of each hexanucleotide at the position $\left[i^{\text {th }} \sim i+5^{\text {th }}\right]$ of the introns. The enrichment of hexanucleotides at position $i$ was analyzed by Fisher's exact test $(p<0.05)$. In case of exon inclusion, two independent tests were performed for exon exclusion and nonregulated exons as follows. The enrichment of the hexanucleotides at position $i$ of exon inclusion was compared with the number of the same motif at the same position $i$ observed in exon exclusion, and vice versa for exon exclusion.

$$
P_{k}(X \geq q)=\sum_{i=q}^{n} \frac{\left(\frac{p}{i}\right)\left(\frac{N-p}{n-i}\right)}{\left(\frac{N}{n}\right)}
$$

where $N$ is the total number of all hexanucleotides found in all exons (i.e., included exons, excluded exons and non-regulated exons), $n$ is the number of all hexanucleotides found in the exons of interest (i.e., exon inclusion OR exclusion), $p$ is the total number of a specific hexanucleotides found in all exons, $q$ is the number of a specific hexanucleotides found in the exons of interest.

\section{KEGG pathway analysis}

The enrichment of genes for KEGG pathways was analyzed through Fisher's exact test $(p<0.05)$ as follows.

$$
P_{k}(X \geq q)=\sum_{i=q}^{n} \frac{\left(\frac{p}{i}\right)\left(\frac{N-p}{n-i}\right)}{\left(\frac{N}{n}\right)}
$$

where $N$ is the total number of genes mapped onto the entire KEGG pathways, $n$ is the number of genes mapped onto the KEGG pathway of interest, $p$ is the total number of exon variants mapped onto the entire KEGG pathways, $q$ is the number of exon variants mapped onto the KEGG pathway of interest.

\section{TAC-induced cardiac hypertrophy}

The 8 weeks old male (C57BL/6J) mice (body weight 28-33 g) were used. Cardiac hypertrophy was induced by transverse aortic constriction (TAC) operation under anesthesia with intraperitoneal injection of avertin, 2-2-2 tribromoethanol (Sigma, USA) dissolved in tert-amyl alcohol (Sigma, USA). The procedure of operation was followed as previously described (Cha et al., 2008). As a control group, sham operation (same procedure except for tying) was done. One week after the operation, mice were sacrificed, and hearts were removed and stored in deep freezer at $-80^{\circ} \mathrm{C}$. Validation of cardiac hypertrophy model was performed by the measurement of heart weight and body weight ratio and quantification for mRNA expression of hypertrophy markers (ANP and BNP; Fig. 3A).

\section{Quantitative real-time PCR (qRT-PCR)}

First-strand cDNA was synthesized from $2 \mu \mathrm{g}$ of total RNA with Random hexamer using Omniscript ${ }^{\circledR}$ reverse transcription (Qiagen, USA) according to the manufacturer's instruction. qRTPCR assays were followed as previously described (Hong et al., 2008). Briefly, qRT-PCR assays were performed using TOPreal $^{T M}$ qPCR premix (Enzynomics, Korea) under the following two-step conditions: denaturation at $95^{\circ} \mathrm{C}$ for $15 \mathrm{~s}$ followed 
A

\begin{tabular}{|c|c|c|c|c|}
\hline 500bp & $500 \mathrm{bp}$ & 500bp & $500 \mathrm{bp}$ & : Exon variants (included/excluded) \\
\hline R1 & R2 & R3 & R4 & $\begin{array}{l}\text { 血 : Upstream exon } \\
\text { 目: Downstream exon }\end{array}$ \\
\hline
\end{tabular}
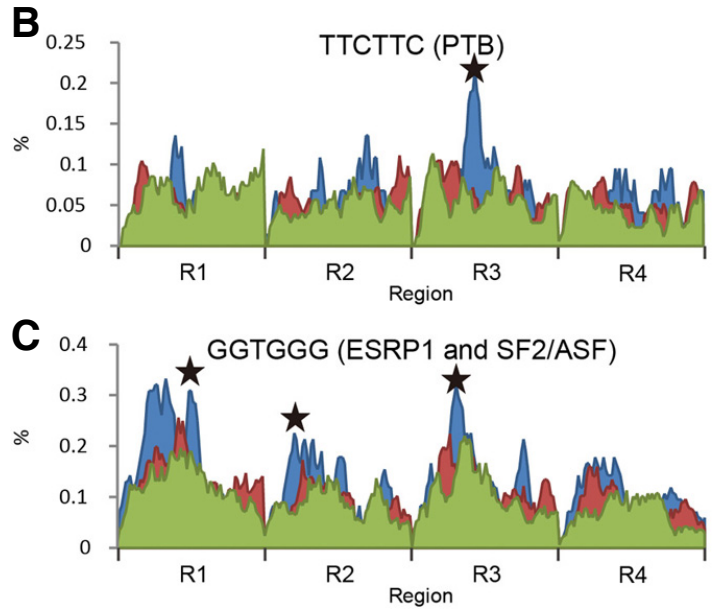

by annealing and extension at $60^{\circ} \mathrm{C}$ for $40 \mathrm{~s}$, for a total of 40 cycles. The $18 \mathrm{~S}$ transcript was used as an endogenous reference to assess the relative level of mRNA transcript. The following primers were used:

\section{ANP, 5'-TCGTCTTGGCCTTTTGGC-3' (sense) and 5'-TCCAGGTGGTCTAGCAGGTTCT-3' (antisense) BNP, 5'-AGGGAGAACACGGCATCATT-3' (sense) and 5'-GACAGCACCTTCAGGAGAT-3' (antisense) 18S, 5'-TTCTGGCGAACGGTCTAGACAAC-3' (sense) and 5'-CCACTGGTCTTGGTGTGCTGA-3' (antisense)}

\section{Western blot}

Frozen mouse hearts were homogenized in liquid nitrogen using a mortar and pestle. Homogenized heart powder was lysed in $1 \%$ SDS lysis buffer containing protease inhibitor cocktail, $\mathrm{Na}_{3} \mathrm{VO}_{4}$ and $\mathrm{NaF}$. Proteins were estimated by using $\mathrm{BCA}$ assay kit (Thermo scientific) and $30 \mu \mathrm{g}$ of proteins were resolved electrophoretically on $10 \%$ SDS polyacrylamide gels, transferred to PVDF membrane. The blots were probed with following antibodies and detected by chemiluminescent substrate (Thermo scientific) and Biomolecular imager, ImageQuant LAS 4000 mini (GE). The band intensities were quantified using NIH ImageJ software.

The following primary antibodies were used for Western blot analysis: ESRP1 (210-301-B89) was purchased from Rockland. PTB, SF2/ASF, SRp30c, and SRp40 antibodies were kind gifts from Dr. Hai-Hong Shen (Gwangju Institute of Science and Technology, Korea).

\section{RESULTS}

Exon variants in cardiac hypertrophy

Our previous study showed the transcriptomic signatures of cardiac hypertrophy and identified hundreds of exon variants that were included or excluded (Song et al., 2012). In the present study, the putative motifs that recruit splicing factors and regulate alternative splicing during cardiac hypertrophy were investigated. For this study, we first collected 3,345 and 3,101
Fig. 2. Positional information for enriched intronic motifs associated with pressure-overload cardiac hypertrophy. (A) A schematic view of the intronic regions used in the analysis. (B, C) Positional frequency of representative enriched intronic motifs (R1-R4 on the $x$-axis indicates the 70 bp adjacent to each exon). The splicing factors predicted to bind to the indicated intronic motifs are shown in parentheses. The black star symbols represent the positions of the significantly enriched intronic motifs. Additional information on the intronic motifs is shown in Supplementary Table 2. included exons and excluded exons, respectively, and then identified 4,125 that were not regulated $(p>0.5)$ during pressure-overload cardiac hypertrophy (See "Materials and Methods"). The distributions of alternative splicing types for the exons of interest (i.e., included exons, excluded exons and non-regulated exons) are shown in Supplementary Fig. 1. The results indicate that alternative promoter $\left(p=7.8 \times 10^{-11}\right)$, alternative termination $\left(p=3.7 \times 10^{-22}\right)$ and cassette exons $(p=$ 0.049 ) are differentially distributed between exon exclusion and exon inclusion.

The exon variants were characterized in terms of their nucleotide composition, length, and the distribution of 5'- or 3' UTRs. As shown in Supplementary Fig. 2A, the nucleotide composition was somewhat distinct across the exon variants; an even distribution of $A, T, G$, and C (ranging from $24 \%$ to $25 \%$ ) was observed in the non-regulated exons, whereas a significantly biased distribution of GC ( 28\%) and AT ( $26 \%)$ was observed in included exons and excluded exons, respectively $(p=2.2 \times$ $10^{-16} ; X^{2}$-test). Interestingly, the distribution of $5^{\prime}$ UTRs and $3^{\prime}$ UTRs in the included exons and excluded exons exhibited an obvious dichotomous pattern between; exons containing 5' UTRs were more likely to be included, whereas exons containing $3^{\prime}$ UTRs tended to be excluded during pressure-overload cardiac hypertrophy (Supplementary Fig. 2B). Longer exons were more likely to be excluded; however, no significant difference was observed in median length across all exons (Supplementary Fig. 2C).

\section{Characterization of putative exonic motifs}

The biased distribution of GC/AT between included and excluded exons and the dichotomous patterns of $5^{\prime}$ UTRs and $3^{\prime}$ UTRs suggest specific architectures (e.g., motifs) that recruit specific splicing factors for either exon inclusion or exon exclusion. To predict the putative motifs in the exon variants, we searched for motifs composed of hexanucleotides that were significantly enriched in an inclusion- or exclusion-specific manner. We found that, among all possible hexanucleotides (i.e., $4^{6}$ $=4,096), 29$ and 24 hexanucleotides were abundantly distributed in excluded exons and included exons, respectively 


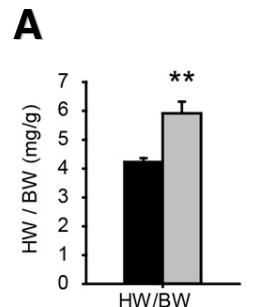

B

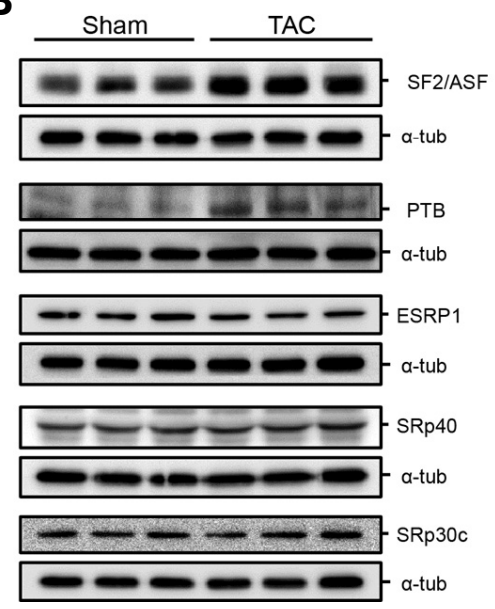

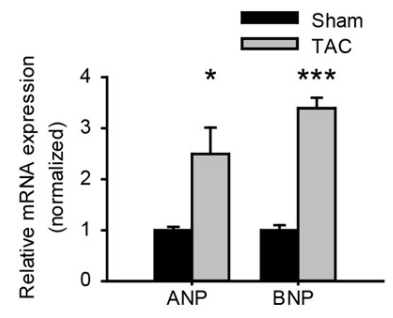

.

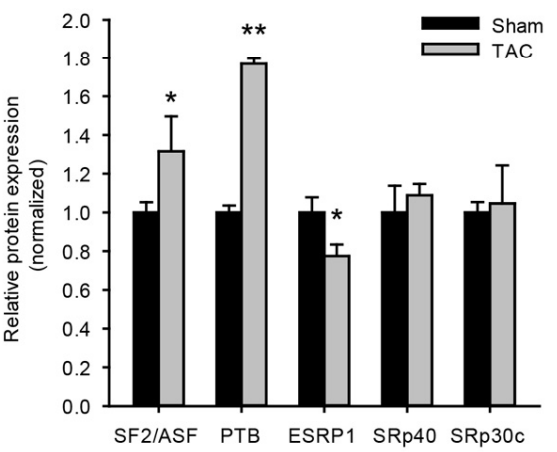

Fig. 3. Protein expression levels of the splicing factors bound to the putative motifs in pressure-overload cardiac hypertrophy. (A) Heart weight/body weight ratio (HW/BW) at 1 week after Sham or TAC operation (left panel). The relative expression levels of the hypertrophic markers in the mouse models were determined by qRT-PCR (right panel). The differences are statistically significant at ${ }^{*} p<0.05,{ }^{* *} p<0.01$ and ${ }^{* * *} p<0.001$ (The student $t$-test). Bars repre-sent means $\pm \mathrm{SD}$ $(n=3)$. (B) The relative protein expression levels of the putative splicing factors bound to the identified intronic motifs were measured by Western blotting. Bars represent means \pm $\mathrm{SD}(\mathrm{n}=3)$. The differences are statistically significant at ${ }^{*} p<0.05,{ }^{* *} p<0.01$ (The student $t$-test).
(Supplementary Table 1). Consistent with the high AT-content pattern observed in the excluded exons (Supplementary Fig. $2 \mathrm{~A})$, motifs with a high proportion of AT were enriched in excluded exons compared to included exons or non-regulated exons (Fig. 1A). The enrichment of AT-rich motifs implies that AT-rich elements (AREs) contribute to the instability of mRNA in the 3' UTRs (Barreau et al., 2005). We also found that 'alternative terminal exons', defined by the 'knownAlt' track of the UCSC genome browser, and $3^{\prime}$ UTRs were significantly enriched in the exons containing AT-rich motifs $(p<0.05)$, implying the removal of AREs in $3^{\prime}$ UTRs (Fig. 1C and Supplementary Table 1). In contrast, motifs with high GC content were observed in included exons (Fig. 1B and Supplementary Table 1). Consistent with the high proportion of $5^{\prime}$ UTRs observed in the included exons, $5^{\prime}$ UTRs participated in the inclusion of exons with anchoring GC-rich motifs (Fig. 1D). The exon inclusion capability of $5^{\prime}$ UTR content does not appear to be related to an alternative promoter, as no known alternative promoters were significantly enriched (Supplementary Table 1). Rather, it seems to be related to intron retention (e.g., $p$-value $=8.19 \times 10^{-5}-6.16$ $\times 10^{-8}$ for GGCCGC, GGCGGC, and CGGCGG; Fisher's exact test). In light of previous reports that exon borders with higher GC content tended to retain introns (Galante et al., 2004; Wong et al., 2013), this result suggests that the GC-rich motifs located in the $5^{\prime}$ UTRs might be strongly related to intron retention during cardiac hypertrophy.

In summary, we found that exons that contain a GC-rich motif in the $5^{\prime}$ UTRs were significantly included and that AT-rich exons with $3^{\prime}$ UTRs were highly excluded during pressure-overload cardiac hypertrophy. These findings also suggest possible roles for GC-rich and AT-rich motifs in intron retention and exclusion of ARE, respectively.
Enriched intronic motifs associated with exon variants

Similar to what was observed in the exonic motif analysis, the enriched intronic motifs associated with exon variants were also investigated. Due to the importance of proximity to exons, the distribution of all possible hexanucleotides within the 500 base pairs (bp) flanking the intronic sequences (i.e., R1-R4) were analyzed (Fig. 2A). We found 13 and 7 motifs with strong positional bias within the $70 \mathrm{bp}$ adjacent to the introns of excluded exons and included exons, respectively (Figs. $2 \mathrm{~B}$ and $2 \mathrm{C}$, and Supplementary Table 2). Interestingly, R1 was the most important region, anchoring eight motifs $(52 \%)$ of the excluded exons. For the included exons, the $\sim 20$ bp upstream of the exons (i.e., R2) were likely to be critical for the binding of splicing factors.

For intronic motifs, we found a correlation between G-rich motifs and intron length. Introns containing G-rich motifs were much shorter than introns containing other motifs. The average intron size in the mouse ranges from 3.8 to $5 \mathrm{~kb}$ (Taft et al., 2007; Waterston et al., 2002), whereas the length of the introns upstream and downstream of the exon variants containing Grich motifs, such as GGAGGG, were less than $1 \mathrm{~kb}$, suggesting a role for these G-rich motifs in the exclusion of exons flanking short introns (Supplementary Fig. 3). Since ESRP1, SF2/ASF, SRp30c, and SRp40 were reported to bind to G-rich motifs (such as GGTGGG, GGAGAG, and GGAGGG) (Supplementary Table 2), we experimentally examined the expression level of splicing factors in Sham/TAC-operated heart sample and found the differential expression of SF2/ASF and ESRP1 (Fig. 3). Grich intronic element at $5^{\prime}$ splice site was known to regulate alternative splicing of thyroid hormone receptor by SF2/ASF (Hastings et al., 2001) and we also found the significantly enriched G-rich motif at 5' splice site along with significantly increased level of SF2/ASF during cardiac hypertrophy. G-rich motifs are also involved in the regulation of alternative splicing by ESRP (Dittmar et al., 2012; Warzecha et al., 2010). Of the 
A Exonic motifs

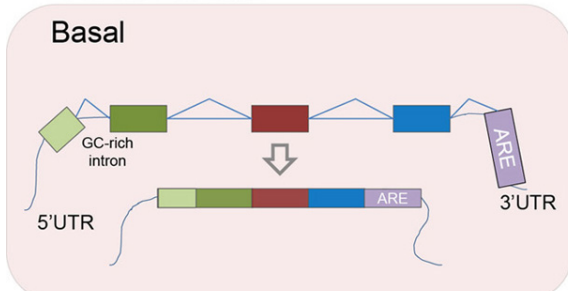

B Intronic motifs

Basal

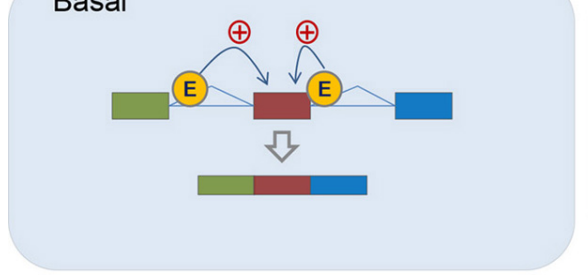

Cardiac hypertrophy

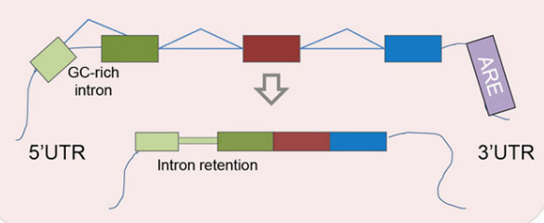

Cardiac hypertrophy

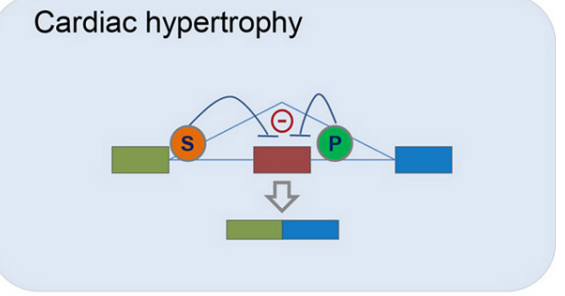

Fig. 4. Schematic diagram showing the representative characteristics of exon variants in cardiac hypertrophy. (A) In cardiac hypertrophy, the exonic motifs in the exon variants lead to retention of $\mathrm{GC}$-rich introns in the mRNA. Conversely, AT-rich elements (AREs) are excluded, making mRNAs more stable. (B) Due to the intronic motifs associated with exon variants, the differential expression of the splicing factors influence exon exclusion or inclusion in cardiac hypertrophy.

$$
\text { E ESRP1 S SF2/ASF P PTB }
$$

G-rich motifs, GTGGG-containing motifs (i.e., GGTGGG and GTGGGG) were significantly enriched in multiple sites for R1 and R3 of 57 exons, which were highly involved in cellular movement ( $p=7.62 \times 10^{-5}$; Fisher's exact test). ESRP is known to bind to TGG motifs, which are located within these GTGGG motifs, and play an important role in cellular movements such as cell adhesion, cell polarity, and cell migration (Warzecha et al., 2010). Interestingly, the GTGGG motifs were found along with TGCCTG motifs in the adjacent 50 bp of R2 $\left(p=1.49 \times 10^{-4}\right)$, suggesting close interplay between these two motifs in exon exclusion. The co-occurrence of TGCCTG at the $3^{\prime}$ splicing acceptor of the exons (i.e., R2) and GTGGG at the $5^{\prime}$ splicing donor of excluded exons (i.e., R3) were observed in Shank3, Vps16, Rhbdl1, Arhgef2, Nbeal2, and Brd9 (data not shown). TGC/CTG repeats are known binding motifs for CELF proteins, which are critical splicing factors during cardiac development (Ladd et al., 2001). Besides the G-rich motifs, we also found the significantly increased PTB known to bind to TCTT motif, during cardiac hypertrophy (Das et al., 2007).

Taken together, we predicted that intronic motifs were significantly enriched at specific positions in introns, and experimentally confirmed the expression of the splicing factors that bind to these motifs. The role of ESRP1 is notable, inducing the exclusion of exons flanking a short intron in genes involved in cellular movement.

\section{DISCUSSION}

During pressure overload cardiac hypertrophy, the heart undergoes rapid enlargement, remodeling and irreversible maladaptation accompanied by dramatically increased energy consumption due to rapid cardiac growth and the synthesis of cellular building blocks. Therefore, systematic cellular and metabolic transitions are necessary to meet the substantial demands during cardiac remodeling. Even though the quantitative signatures of pathological cardiac hypertrophy have been well studied (Park et al., 2011; Song et al., 2012), the qualitative changes during cardiac remodeling have remained to be investigated. Here, we show the evidence for the association of alternative splicing with pressure-overload hypertrophy as follows: 1) GCrich exonic motifs regulate intron retention in 5' UTRs and AT- rich exonic motifs are involved in exclusion of the AT-rich elements in 3' UTRs during cardiac hypertrophy (Figs. 1 and 4, and Supplementary Table 1);2) 20 putative splicing factor binding motifs enriched in intronic regions were identified (Fig. 2 and Supplementary Table 2); 3) Splicing factors such as PTB and ESRP1 that are known to bind to the putative motifs were differentially expressed during cardiac hypertrophy (Fig. 3); 4) Collectively, the splicing regulatory elements are involved in posttranscriptional regulation via the $3^{\prime}$ or $5^{\prime}$ UTRs or by recruiting splicing factors during cardiac hypertrophy (Fig. 4).

As summarized in Fig. 4A, alternative splicing for both $5^{\prime}$ and $3^{\prime}$ UTRs may play an important role in regulating translation during cardiac remodeling. The observed retention of introns in $5^{\prime}$ UTRs is important, because the mechanism could be involved in post-transcriptional regulation by inserting regulatory elements such as internal ribosome entry site (IRES) and Gquadruplex into mRNA. According to the previous reports, the intron retention led to the acquisition of IRES in Zeb2 and to the insertion of G-quadruplex in p53 (Beltran et al., 2008; Marcel et al., 2011). In the case of alternative splicing for $3^{\prime}$ UTR, we observed the removal of AREs that make the mRNA unstable. These findings suggest that post-transcriptional regulation by alternative UTRs might increase mRNA stability, thereby maximizing protein synthesis without significantly increasing transcription. Based on the rapid increase in heart volume during cardiac hypertrophy, this mechanism is an excellent way to adapt against urgent cardiac overload.

As shown in Fig. 4B, we found the altered expression of splicing factors that bind to the intronic motifs. Among them, PTB and ESRP1 are already known to be involved in alternative splicing of smooth muscle and metastasis in cancer, respectively (Gooding et al., 2013; Reinke et al., 2012; Yae et al., 2012), but their roles in cardiac hypertrophy are not known. They likely bind to the G-rich regions and are widely distributed nearby exons excluded (Supplementary Table 2). Warzecha et al. (2010) reported that UGG is a core motif of ESRP1 and the motif located in upstream intron possibly promotes exon exclusion, whereas the motifs in downstream of the regulated exon enhance exon inclusion. Their following study on the ESRP1 binding motifs using SELEX-Seq predicted the enriched GUrich motifs in the flanking upstream (R2) of the excluded exons. 
For the included exons, they found the motifs significantly enriched in the distal upstream (R1) and flanking downstream (R3). During cardiac hypertrophy, the decreased level of ESRP1 might result in the weak binding at R1 and R3 as reported recently (Dittmar et al., 2012).

Intronic GU-rich repeats at $5^{\prime}$ splice site are also known to be the binding region for SF2/ASF and hnRNP H (Arrisi-Mercado et al., 2004; Hastings et al., 2001). Similarly, we also found the significantly increased level of SF2/ASF during cardiac hypertrophy. However, the detailed mechanistic relationship between the differential expression of the splicing factors (e.g., ESRP1 and SF2/ASF) and the G-rich motifs remains to be elucidated, as the splicing factors usually play roles as both enhancer and repressor depending on the position, concentration and the location of the branchpoint (Lim et al., 2011; Taggart et al., 2012; Zuo and Manley, 1993).

Consistent with the known role of ESRP1 in the alternative splicing of genes involved in cellular movement such as cell adhesion, polarity and migration (Warzecha et al., 2010), we also identified 57 genes with the ESRP1 binding motif that are involved in cellular movement. The significantly decreased expression of ESRP1 (Fig. 3) also suggests the importance of ESRP1 in alternative splicing during cardiac hypertrophy. We also identified a significant co-occurrence of the binding motifs for ESRP1 and CELF. However, the expression of the gene encoding CELF did not change during cardiac hypertrophy (data not shown). Although there was no change in the expression of CELF genes (e.g., Cugbp1, Cugbp2, Brunol4, and Brunol5), CELF expression can possibly be regulated post-transcriptionally through microRNAs (Kalsotra et al., 2010).

Here, we report the splicing regulatory elements and the putative splicing factors that may play important roles for pathological cardiac hypertrophy. The molecular mechanisms for alternative splicing regulated by the splicing regulatory elements and the functional roles of the individual splicing factors identified from this study will await the future molecular studies.

Note: Supplementary information is available on the Molecules and Cells website (www.molcells.org).

\section{ACKNOWLEDGMENTS}

This work was supported by the Bio \& Medical Technology Development Program of the National Research Foundation (NRF) funded by the Ministry of Science, ICT \& Future Planning (NRF-2013M3A9A7046297, NRF-2012R1A1A2043217) and the 2013 GIST Systems Biology Infrastructure Establishment Grant. We thank Dr. Hai-Hong Shen (Gwangju Institute of Science and Technology, Korea) for the generous gifts of PTB, SF2/ASF, SRp30c, and SRp40 antibodies.

\section{REFERENCES}

Ames, E.G., Lawson, M.J., Mackey, A.J., and Holmes, J.W. (2013). Sequencing of mRNA identifies re-expression of fetal splice variants in cardiac hypertrophy. J. Mol. Cell Cardiol. 62, 99-107.

Arrisi-Mercado, P., Romano, M., Muro, A.F., and Baralle, F.E. (2004). An exonic splicing enhancer offsets the atypical GU-rich $3^{\prime}$ splice site of human apolipoprotein A-II exon 3. J. Biol. Chem. 279, 39331-39339.

Barreau, C., Paillard, L., and Osborne, H.B. (2005). AU-rich elements and associated factors: are there unifying principles? Nucleic Acids Res. 33, 7138-7150.

Beltran, M., Puig, I., Pena, C., Garcia, J.M., Alvarez, A.B., Pena, R., Bonilla, F., and de Herreros, A.G. (2008). A natural antisense transcript regulates Zeb2/Sip1 gene expression during Snail1induced epithelial-mesenchymal transition. Genes Dev. 22, 756769.
Cha, H., Kim, J.M., Oh, J.G., Jeong, M.H., Park, C.S., Park, J., Jeong, H.J., Park, B.K., Lee, Y.H., Jeong, D., et al. (2008). PICOT is a critical regulator of cardiac hypertrophy and cardiomyocyte contractility. J. Mol. Cell Cardiol. 45, 796-803.

Das, D., Clark, T.A., Schweitzer, A., Yamamoto, M., Marr, H., Arribere, J., Minovitsky, S., Poliakov, A., Dubchak, I., Blume, J.E., et al. (2007). A correlation with exon expression approach to identify cis-regulatory elements for tissue-specific alternative splicing. Nucleic Acids Res. 35, 4845-4857.

Dittmar, K.A., Jiang, P., Park, J.W., Amirikian, K., Wan, J., Shen, S., Xing, Y., and Carstens, R.P. (2012). Genome-wide determination of a broad ESRP-regulated posttranscriptional network by high-throughput sequencing. Mol. Cell. Biol. 32, 1468-1482.

Erkelenz, S., Mueller, W.F., Evans, M.S., Busch, A., Schoneweis, K., Hertel, K.J., and Schaal, H. (2013). Position-dependent splicing activation and repression by SR and hnRNP proteins rely on common mechanisms. RNA 19, 96-102.

Eswaran, J., Horvath, A., Godbole, S., Reddy, S.D., Mudvari, P., Ohshiro, K., Cyanam, D., Nair, S., Fuqua, S.A., Polyak, K., et al. (2013). RNA sequencing of cancer reveals novel splicing alterations. Sci. Rep. 3, 1689.

Fairbrother, W.G., Yeh, R.F., Sharp, P.A., and Burge, C.B. (2002). Predictive identification of exonic splicing enhancers in human genes. Science 297, 1007-1013.

Galante, P.A., Sakabe, N.J., Kirschbaum-Slager, N., and de Souza, S.J. (2004). Detection and evaluation of intron retention events in the human transcriptome. RNA 10, 757-765.

Gooding, C., Edge, C., Lorenz, M., Coelho, M.B., Winters, M., Kaminski, C.F., Cherny, D., Eperon, I.C., and Smith, C.W. (2013) MBNL1 and PTB cooperate to repress splicing of Tpm1 exon 3. Nucleic Acids Res. 41, 4765-4782.

Hastings, M.L., Wilson, C.M., and Munroe, S.H. (2001). A purinerich intronic element enhances alternative splicing of thyroid hormone receptor mRNA. RNA 7, 859-874.

Hirose, T., Ideue, T., Nagai, M., Hagiwara, M., Shu, M.D., and Steitz, J.A. (2006). A spliceosomal intron binding protein, IBP160, links position-dependent assembly of intron-encoded box C/D snoRNP to pre-mRNA splicing. Mol. Cell 23, 673-684.

Hong, S.E., Park, I., Cha, H., Rho, S.H., Park, W.J., Cho, C., and Kim, D.H. (2008). Identification of mouse heart transcriptomic network sensitive to various heart diseases. Biotechnol. J. 3, 648-658.

Huh, G.S., and Hynes, R.O. (1994). Regulation of alternative premRNA splicing by a novel repeated hexanucleotide element Genes Dev. 8, 1561-1574.

Kalari, K.R., Rossell, D., Necela, B.M., Asmann, Y.W., Nair, A., Baheti, S., Kachergus, J.M., Younkin, C.S., Baker, T., Carr, J.M., et al. (2012). Deep sequence analysis of non-small cell lung cancer: integrated analysis of gene expression, alternative splicing and single nucleotide variations in lung adenocarcinomas with and without oncogenic KRAS mutations. Front Oncol. 2, 12.

Kalsotra, A., Wang, K., Li, P.F., and Cooper, T.A. (2010). MicroRNAs coordinate an alternative splicing network during mouse postnatal heart development. Genes Dev. 24, 653-658.

Kim, E., Goren, A., and Ast, G. (2008). Alternative splicing and disease. RNA Biol. 5, 17-19.

Kuroyanagi, H., Watanabe, Y., Suzuki, Y., and Hagiwara, M. (2013) Position-dependent and neuron-specific splicing regulation by the CELF family RNA-binding protein UNC-75 in Caenorhabditis elegans. Nucleic Acids Res. 41, 4015-4025.

Ladd, A.N., Charlet, N., and Cooper, T.A. (2001). The CELF family of RNA binding proteins is implicated in cell-specific and developmentally regulated alternative splicing. Mol. Cell. Biol. 21, 1285-1296.

Lim, K.H., Ferraris, L., Filloux, M.E., Raphael, B.J., and Fairbrother, W.G. (2011). Using positional distribution to identify splicing elements and predict pre-mRNA processing defects in human genes. Proc. Natl. Acad. Sci. USA 108, 11093-11098.

Marcel, V., Tran, P.L., Sagne, C., Martel-Planche, G., Vaslin, L., Teulade-Fichou, M.P., Hall, J., Mergny, J.L., Hainaut, P., and Van Dyck, E. (2011). G-quadruplex structures in TP53 intron 3: role in alternative splicing and in production of p53 mRNA isoforms. Carcinogenesis 32, 271-278.

Park, J.Y., Li, W., Zheng, D., Zhai, P., Zhao, Y., Matsuda, T., Vatner S.F., Sadoshima, J., and Tian, B. (2011). Comparative analysis of mRNA isoform expression in cardiac hypertrophy and deve- 
lopment reveals multiple post-transcriptional regulatory modules. PLoS One 6, e22391.

Reinke, L.M., Xu, Y., and Cheng, C. (2012). Snail represses the splicing regulator epithelial splicing regulatory protein 1 to promote epithelial-mesenchymal transition. J. Biol. Chem. 287, 36435-36442.

Ren, S., Peng, Z., Mao, J.H., Yu, Y., Yin, C., Gao, X., Cui, Z., Zhang, J., Yi, K., Xu, W., et al. (2012). RNA-seq analysis of prostate cancer in the Chinese population identifies recurrent gene fusions, cancer-associated long noncoding RNAs and aberrant alternative splicings. Cell Res. 22, 806-821.

Song, H.K., Hong, S.E., Kim, T., and Kim, D.H. (2012). Deep RNA sequencing reveals novel cardiac transcriptomic signatures for physiological and pathological hypertrophy. PLoS One 7, e35552.

Taft, R.J., Pheasant, M., and Mattick, J.S. (2007). The relationship between non-protein-coding DNA and eukaryotic complexity. Bioessays 29, 288-299.

Taggart, A.J., DeSimone, A.M., Shih, J.S., Filloux, M.E., and Fairbrother, W.G. (2012). Large-scale mapping of branchpoints in human pre-mRNA transcripts in vivo. Nat. Struct. Mol. Biol. 19, 719-721.

Vencio, R.Z., Brentani, H., Patrao, D.F., and Pereira, C.A. (2004). Bayesian model accounting for within-class biological variability in serial analysis of gene expression (SAGE). BMC Bioinformatics $5,119$.

Warzecha, C.C., Jiang, P., Amirikian, K., Dittmar, K.A., Lu, H., Shen,
S., Guo, W., Xing, Y., and Carstens, R.P. (2010). An ESRPregulated splicing programme is abrogated during the epithelialmesenchymal transition. EMBO J. 29, 3286-3300.

Waterston, R.H., Lindblad-Toh, K., Birney, E., Rogers, J., Abril, J.F., Agarwal, P., Agarwala, R., Ainscough, R., Alexandersson, M., An, P., et al. (2002). Initial sequencing and comparative analysis of the mouse genome. Nature 420, 520-562.

Wong, J.J., Ritchie, W., Ebner, O.A., Selbach, M., Wong, J.W. Huang, Y., Gao, D., Pinello, N., Gonzalez, M., Baidya, K., et al. (2013). Orchestrated intron retention regulates normal granulocyte differentiation. Cell 154, 583-595.

Yae, T., Tsuchihashi, K., Ishimoto, T., Motohara, T., Yoshikawa, M., Yoshida, G.J., Wada, T., Masuko, T., Mogushi, K., Tanaka, H., et al. (2012). Alternative splicing of CD44 mRNA by ESRP1 enhances lung colonization of metastatic cancer cell. Nat. Commun. 3, 883 .

Yamashita, A., Shichino, Y., Tanaka, H., Hiriart, E., Touat-Todeschini, L., Vavasseur, A., Ding, D.Q., Hiraoka, Y., Verdel, A., and Yamamoto, M. (2012). Hexanucleotide motifs mediate recruitment of the RNA elimination machinery to silent meiotic genes. Open Biol. 2, 120014

Zhou, J., Zheng, X., and Shen, H. (2012). Targeting RNA-splicing for SMA treatment. Mol. Cells 33, 223-228.

Zuo, P., and Manley, J.L. (1993). Functional domains of the human splicing factor ASF/SF2. EMBO J. 12, 4727-4737. 